\title{
Retractorless Surgery of Vascular Lesions
}

\author{
Thomas Kretschmer, Christian Heinen, \\ and Thomas Schmidt
}

\subsection{Introduction}

The introduction of the operating microscope enabled modern neurosurgery. For usually deepseated vascular lesions and confining, overhanging brain parenchyma walls, self-retaining retractors were necessary to enable open and safe dissection corridors. However, retractors also exert secondary pressure effects and can limit the degree of manual freedom [1].

Since longer a rigid retractor-free technique for cerebral surgery in general and cerebrovascular pathology in particular has been propagated. Professor Yasargil's published comment on Kivisaari et al.'s 2000 paper on sequelae of retractor use [2] merits reiteration: "most prominent finding supports that one-third $(36 \%)$ of the patients is related with brain retractor. All effort to reduce the use of brain retractor such as wide splitting of the sylvian fissure, release the CSF from the basal cisterns, and the use of mannitol, should be done with also the attention to appreciate all arteries and veins during dissection."

Despite lower surgical case rates after publication and unjustified generalization of the ISAT 2002 results [3], cerebrovascular surgery developed substantially and in proficient hands at many centers changed toward considerably more

T. Kretschmer $(\bowtie) \cdot$ C. Heinen · T. Schmidt Department of Neurosurgery, Evangelisches Krankenhaus Oldenburg, Oldenburg, Germany focused, efficient, and extremely tissue sparing surgery [4]. As was put forward by the Spetzler group discussion, the 3-year BRAT results have also pointed out that if the surgeon avoids using the rigid retraction during complex aneurysm surgery, it will avoid adjacent brain injury [4].

To date a growing community of neurosurgeons thus adopted a retractorless dissection technique by making full use of neurosurgeries' current armamentarium, merging all the long known knowledge and tricks with the tools of modern microsurgery. Implementation of a bimanual dissection technique without rigid fixation but rather intensive use of microscope mouthpiece and foot control enables us to work within very confined spaces perfectly safe.

\subsection{Brain Retraction}

Brain retraction can produce high local pressure on the brain and then reduce the blood flow locally, and some surgeon said that the retractor could hide the anatomy under the retractor. If the brain swelling, sometimes the retractor also directly injures and sometimes cut through the brain. Narrow rigid retractor blades on already edematous tissue lead to even higher tissue pressures (e.g., incising retractor edges). Brain retraction for several minutes to hours can exert permanent pressure effects. Some of these effects can be demonstrated on post-op MRI [5]: high 
signal was demonstrated in one-third of clipped aneurysm cases during follow-up (36/101 cases). They were located at the basal frontotemporal area of the approach in aneurysm clippings of the anterior circulation. These signals could be traced 2-6 years after surgery and thus were interpreted as signs of damage.

\subsection{With Fixed Retractors}

Working with self-retaining, rigid retractors enables and favors a different style of dissection and is possible without the use of a microscope mouthpiece. The retractors not only create an open view on the exposed pathology but also free the hands to easily move out of the operative site to adjust for zoom, focus, or microscope headpiece position or receive and change instruments. A set retractor usually opens a funnel-shaped straight corridor from the surface to the depth to allow for an unimpeded view to the bottom of the created corridor. During the course of surgery, retractors are frequently reset. Long-standing brain retraction or retraction on swollen or "red and angry" brain can induce direct and instantly visible parenchyma damage. Expert microsurgeons make a point that proper retractor setting never exerts pressure but only is placed on the brain surface as shelter.

\subsection{Without Fixed Retractors}

In principle of retractorless surgery, dynamic and constantly alternating mini-retraction is applied by the microinstruments to proceed along natural sulcal and fissural trails until the pathology is reached. So, in its true sense, surgery is not retraction-free but free of fixed retraction. Craniotomies are planned in a way to create corridors by gravity even before dissection starts. The combined measures of optimal positioning, "custom-tailored" focused craniotomy with drilling on the cranial base side, adequate anesthesia for slack brain, and CSF drainage via the cisterns plus meticulous bloodless dissection most of the time opens a dissection corridor that will stay open on its own without additional measures (see figures for approach examples). So, there is no need to pull parenchyma out of the way. The created dissection corridors remain open. We are sure that anyone who made a "successful" transition from the use of fixed retraction to retractorless also experienced that his way to handle brain and vessels developed in equal measure, as the need for more effective movement arises, and back and forth movements are discouraged.

\subsection{Elements}

\subsubsection{Operative Field (Fig. 30.1)}

The surgeons' hands need to be unrestricted and free at all times.

For this the operative field should be tidy and not overcrowded by appliances, tubing, and cables. We prefer to have nothing in the field that could potentially interfere with our hands. Everything not on the side opposite to the surgeon like a Leyla bar is attached to two adjustable rods attached to the head clamp (Mayfield system). We prefer to set up the rods like a frame around the head resembling a tilted V. This V-frame is positioned far below the plane of craniotomy to ensure that the attached soft tissue hooks can be kept low profile and are not spanned out above the craniotomy. Everything

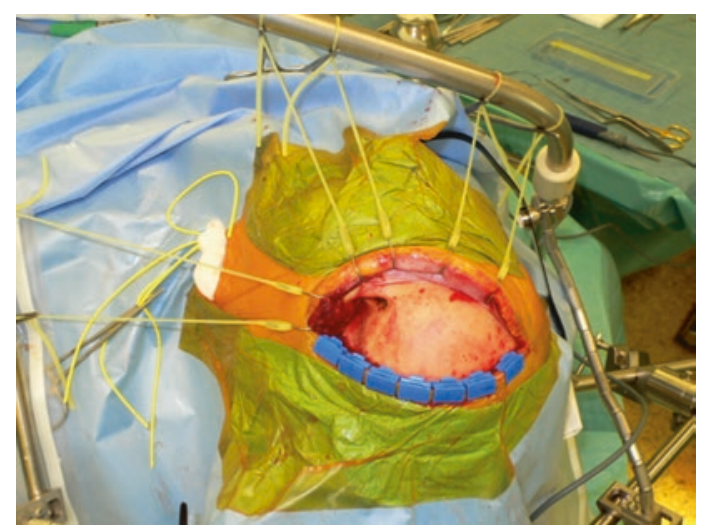

Fig. 30.1 Operative field. The field needs to be set up tidy and kept absolutely clean, and any clutter from cables and extensions should be avoided to keep the space above the craniotomy clear for unrestricted movement of hands 
else attached to the rods will also be fixed in such a manner. With Sugita frames, the system as such implements a ring that is placed following the same principles.

\subsubsection{Microscope}

The use of a microscope is precondition to modern neurosurgery: retractorless neurosurgery relates to intracranial neurosurgery during the microscopic phase of surgery. Retractorless neurosurgery is dependent on microscopes that can be operated with a mouthpiece. It is not possible to perform retractorless microsurgery with microscopes that exclusively need to be repositioned manually. The microscope needs to be especially well balanced if it is worked with a mouthpiece in order to prevent annoying microoscillation after release and engagement of brakes via the mouthpiece.

\subsubsection{Mouthpiece (Fig. 30.2)}

The proper position of the mouthpiece needs to be individually adjusted for every surgeon, and even for every surgeon individually for every case, as the main direction and angle of the microscope head will depend on the approach used. So, for posterior fossa surgery, a far steeper angle in the sagittal plane is used in comparison to the alignment for a supratentorial convexity process.

For adjusting the mouthpiece, the microscope head is brought in the anticipated direction, the pupillary distance is set on the oculars, and the head and eyes are brought in the surgeon's usual position. Then all three locking screws are opened, and the mouthpiece is introduced in the mouth, with contact of the upper plate to the upper teeth and lip; the microscope head brakes are disengaged when the lower mouthpiece plate is released by gentle pressure of the lower lip. By holding the released position with the lower lip, the microscope head will float, if the microscope has been properly balanced (as opposed to pull in one direction if not balanced). Now, it is possible

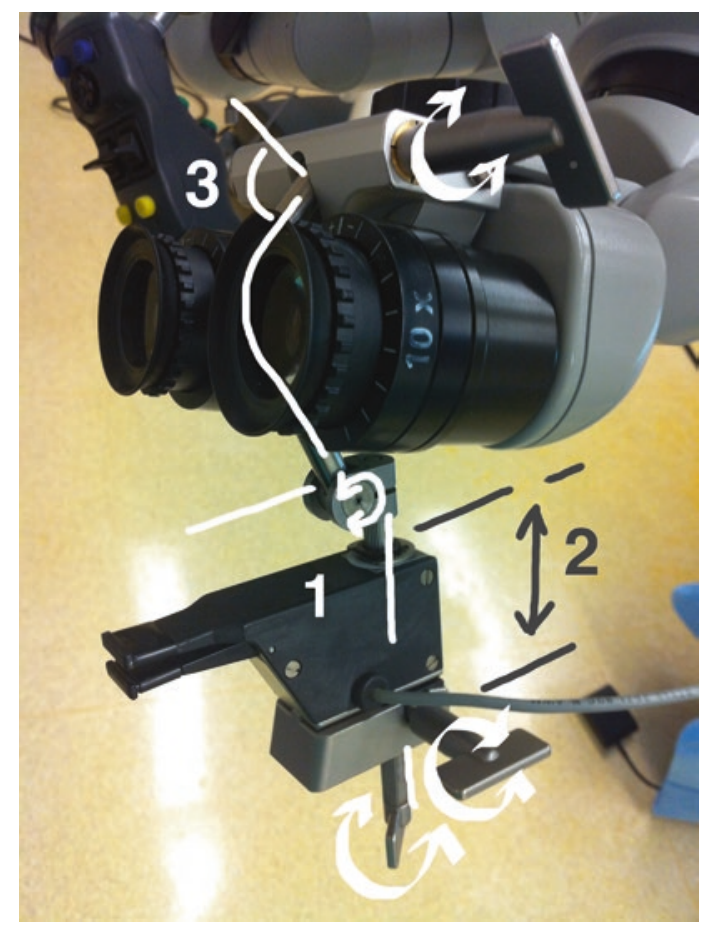

Fig. 30.2 Mouthpiece. Mastering use of the mouthpiece is absolute key to retractorless surgery. A first step is to be able to properly position it in the mouth to not interfere with the accustomed head and eye lens position. Therefore, it can be adapted by changing its position in two planes. The joints marked at 1 and 3 enable movement in an axial plane to bring the piece forward into the mouth. Number 2 marks possible vertical movement. The three screws are loosened, head and eyes are brought in the operative position, and then the mouthpiece is guided into the mouth in a way that the lips can have close contact with the mouthpiece blades

to steer the microscope with the mouth in three planes. Thus, it can be steered to easily follow the focus point of dissection. By slightly changing the distance of the microscope in relation to the surgical plane, the focus can be adapted and finetuned without having to use the focus button of the microscope/foot paddle constantly. By using this technique for subtle focus adaption, the focus change by microscope handle or foot paddle will be only necessary for larger focus changes. Once used to this tool, the involved maneuvers pass off completely "subcortical." This becomes the surgeon's major tool to prevent frequent in and out maneuvers of the surgeons' hands out of the surgical site. It is precondition to establish a real 
flow of movements and improves surgical efficiency immensely, since every movement and instrument maneuver lead to advance dissection one step further without being interrupted by frequent adaption movements. The other merit is true bimanual work in the sense of alternating the "leading" hand (if there is one) in dependence on suitability of current position and not freedom of hand. Coarser adjustments of the microscope by movement via microscope head handles are disengaged and only necessary less frequently. Most coarse adjustments can be executed with a foot pedal (zoom-focus-position of microscope head).

\subsubsection{Foot Pedal (Fig. 30.3)}

The foot pedal has at least two rocker-like pedals that are used for bidirectional zoom and focus. Usually on the pedal frame, there are additional knobs that can be individually set for other functions (photo documentation, light adjustment, video start/stop). There are foot pedals that have

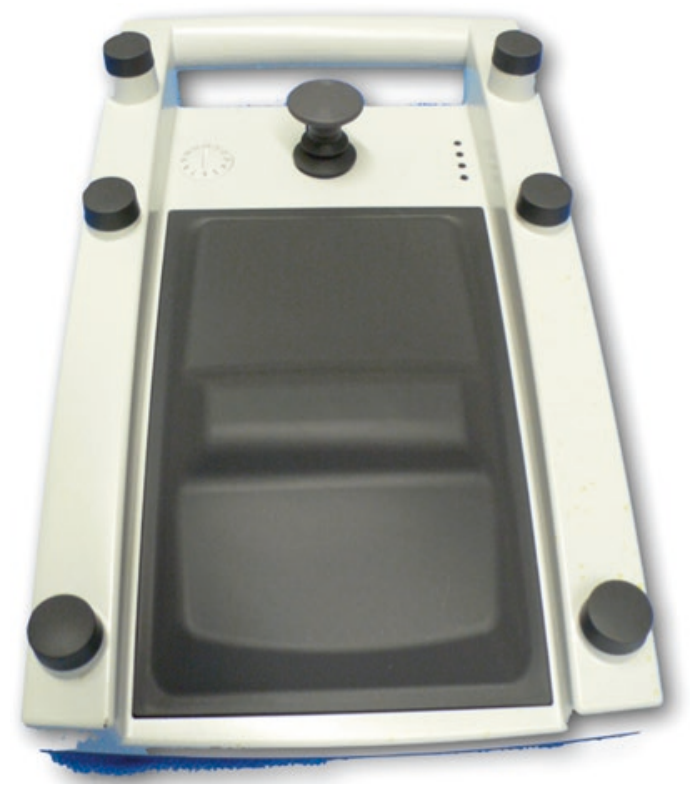

Fig. 30.3 Foot pedal. Foot pedals can serve multiple functions with freely selectable function allocation to several knobs (e.g., photo, video on/off, \pm light, ICG on/off). The joystick steers the microscope head; \pm zoom and \pm focus can be allocated to the left or right side. Wireless function transmission avoids cable clutter an integrated joystick that allows for stepless adjustment of the microscope head in all planes. In interplay with an armrest chair, a wireless pedal avoids cable clutter at the surgeons' feet and eases change of chair position.

\subsubsection{Chair or Other Means of Armrest (Fig. 30.4)}

Resting the forearms or at least the hand by contact of the small finger on the surface to stabilize the hand is a basic microsurgical principle. It eases and enables precise finger and instrument movements. Appropriate chairs with mounted armrests can be used independent from patient positioning and method of head fixation. There are many useful models, depending on the individual preferences.

Several points with regard to positioning of the surgeon are crucial to aid microsurgery as

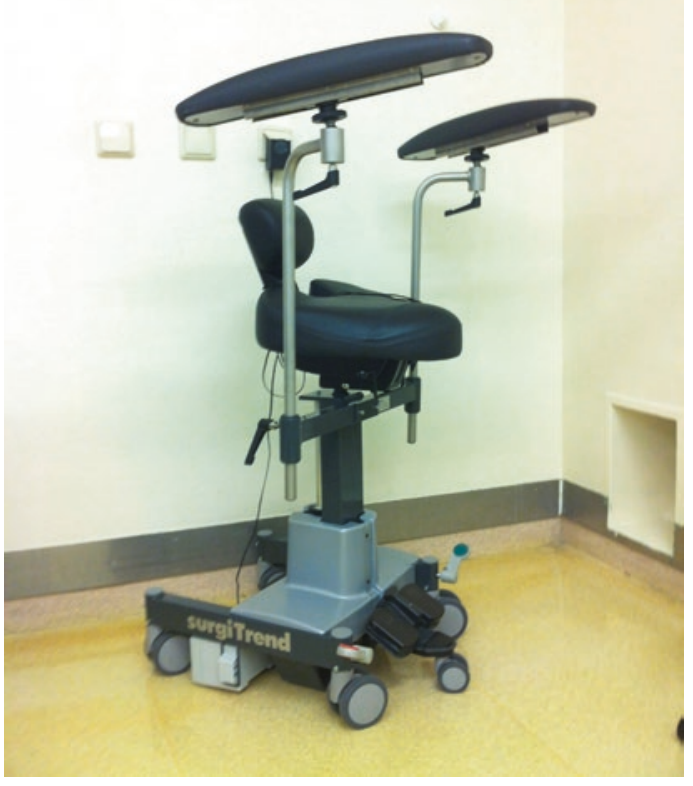

Fig. 30.4 Armrest and chair. Chair and armrests should be adjustable in every plane and height to accustom for all possible surgeon and patient positions. Useful is a function that enables to move the armrests backward and forward. The base should not protrude too much forward in order to avoid interference with foot pedals 
such, and long, concentrated microsurgical work in particular, as well as to improve security of risky maneuvers. The precondition of rested forearms is easier to achieve in a setting where the surgeon works on the surface of an extremity (e.g., reconstructive microsurgical hand surgery, where you can place and rest your hand on an arm table or microsurgical work at the surface of the head). It is not so easy, however, to achieve if working under uncomfortable angles in the depth of the neurocranium. To spend some time to think about the adequate position of the surgeon is absolutely helpful and required to perform long-standing retractorless microsurgical work. It should be comfortable enough to allow for long dissection, it should enable to rest the forearms without compromise, and it should not interfere at all with the head clamp and its extensions. Neurosurgeons tend to bend their neck forward and take up a swan neck posture. This is a small detail that probably only can be prevented if corrected early in the career; it strains the neck muscles and the cervical spine. A great way to ease microsurgical dissection as such and improve microsurgical endurance is proper alignment of the upper body and shoulders. The proper height of flexible armrests of an accordingly multi-adjustable stool can greatly remove strain from the shoulder girdle. For instance, the armrests are usually set much higher if working retrosigmoid in the posterior fossa. The surgeon's shoulder girdle should not be tense at all during the microsurgical phase. Of course, experienced microsurgeons will be able to also perform microsurgical movements with straightened arms, involving also shoulder muscles; but it does not make a lot of sense to also involve the deltoid and trapezius in microsurgery if it can be prevented (e.g., doing $\mathrm{CP}$ angle surgery in an uncomfortable position below the craniotomy level with straight arms to reach far enough). Some of the neurosurgical icons prefer to operate standing with a flexible arm board stand because they will be fast enough to not require a sitting position and rather prefer to be able to frequently change their own standing position in relation to the patient. The principle still remains the same. A standing position, however, excludes comfortable simultaneous use of two or more foot pedal-driven devices (multifunction foot pedal, bipolar, ultrasonic aspirator). When a pedal is used standing, the surgeon completely bears his weight on one leg for the time using the pedal. This can be bothersome, when you make continuous use of one or two pedal devices.

\subsubsection{Cisterns}

The ability to open cisterns fast and tissue sparing is pivotal to open a microsurgical dissection corridor at the beginning of the microsurgical phase of surgery.

Knowledge of appropriate measures to reach the decisive neighboring cistern shortly after craniotomy is thus very important. Reaching the cistern very early is largely connected to precise placement of craniotomy and measures for slack brain to avoid undue pressure on brain parenchyma in order to reach deep.

Additional space is gained by reducing the bone right after craniotomy, before dural opening. Bony eminences or the floor of involved skull base and the inner tabula of respective craniotomy rims are neatly drilled down. Several millimeters at the exposure entrance can make a large difference for the microscopic view in the depth and frequently enable to reach a cistern with no or only minimal and short retraction of brain.

\subsubsection{Anesthesiology}

Good interaction with anesthesiology is precondition to reach the necessary brain relaxation for effortless opening of surgical corridors without the use of fixed retraction. We like to start fast infusion of mannitol after the patient has been prepped and draped to reach its full effect right after craniotomy. Among many other factors, the following anesthesiological competencies are quite essential:

- Awareness for the kind of surgery undertaken.

- Awareness of pathology.

- Awareness of cerebral condition. 
- Awareness of possible hazardous complications and resultant need for instant and adequate response.

- Appropriate maneuvers to slacken the brain.

- Experience with induction of measures to increase brain tolerance to ischemia from intentional temporary clipping of major branches.

\section{As for drugs:}

- Mannitol to dehydrate brain tissue $(1 \mathrm{~g} / \mathrm{kg})$.

- Decadron to minimize edema from pressure effects or other mechanical injuries depending on the individual pathology.

- ICG application.

- Systemic adenosine administration for emergency cardiac standstill.

\subsubsection{Instruments Used}

There are no magic instruments; it is the handling of them. All the usual microinstruments can be used. Some instruments like clip appliers have been especially designed for keyhole approaches with long, narrow shafts and modified clip mechanisms. Lighted instrument tips are the current novelty. A major tool remains the suction device. There is great variance in terms of shape, length, tip design, and fenestration for suction strength regulation.

The sucker is by far more than a mere suction device, and its full potential as dynamic macroand microretractor, tissue dissector, and tissue aspirator is to be discovered. Its potential for stepless suction force control needs to be mastered. Precise control of suction force with the thumb over the opening is absolute key (rolling the thumb back and forward over the fenestration). We prefer a keyhole-teardrop-shaped opening to a circular one as it allows for fine-tuned suction control, especially for cerebrovascular work.

The merit of special instruments as such is completely overrated, as we think every surgeon needs to find out and decide on some basic microinstruments that allow him to manage the bulk of his microsurgical work. Permanent use of, and getting used to, the same instruments is the real key. Consequently, our basic trays might appear quite reduced, and are not stuffed with instruments. We rather open separate instruments according to real need for them (e.g., special curette, plate knife, fish-hooked knife). However, it greatly helps to not wait for instruments, which have to be frequently cleaned during surgery (e.g., bipolar forceps). So, it might make sense to have several of the same type on the table, to not stop the surgical flow abruptly because an instrument is cleaned. In fact, if you work retractorless, and especially during a demanding microsurgical phase, it is quite bothersome, tiring, and also dangerous if you frequently were to wait with an outreached arm for an instrument while being completely focused to the pathology and, at the same time, holding a vessel or part of the brain with your dynamic retraction device at the other hand.

Only very few surgeons are ambidextrous in its true sense, and most of them still will have a more dominant (e.g., mainly cutting) hand. However, if working retractorless, it is very useful if the surgeon is not dependent on using a microscissors or knife exclusively with his dominant hand. A good surgical flow can then be established if a structure that needs to be held, retracted, or cut is handled in that way at the moment where this becomes clear without any more time delay by changing hand. Going back and forth before execution of a maneuver is not efficient (e.g., realize adhesion that just became visible by slight microretraction of tissue needs to be cut, letting loose again, look again, reconfirm several times, and then execute the maneuver). It is obvious that the hand that is the easiest to perform a certain maneuver might be on the right or left in dependence on the hand that performs the microretraction maneuver at the moment.

Think of:

- Removing the bone or sacrifice the skull base bone rather than the brain.

- Using one of the hands as dynamic suction and alternating to the other side so the surgeon may avoid the need of retractor.

- Sucker tip retraction-As microretraction.

- Sucker shaft retraction-As macroretraction.

- Microscissors and bipolar shafts that also allow for soft retraction and temporarily can retract their side of the dissection funnel. 
- Microretraction with instrument tips.

- Use all the fingers efficiently including the use of the thumb to control fenestration.

\subsection{Advantages of Retractorless Dissection Technique}

In combination with the mentioned techniques, we see the following advantages of a retractorfree dissection technique:

- More thought on approach planning, positioning, and trajectory.

- Free surgical field.

- Concentration on manual maneuvers and approach tactics.

- Forced use of mouthpiece and foot pedal, therefore more freedom of hands and less unnecessary in and out maneuvers of the hands.

- No manual readjustment of zoom, focus, and microscope head alignment.

- Thus, improved surgical flow by omission of redundant movements and readjustments.

- Overall streamlined (efficient and effective) surgery.

- Less collateral damage.

\subsection{Learning and Transition}

Retractorless surgery goes completely along with the principles of "safe and simple surgery" outlined by Hernesniemi and the Helsinki group [6]. The novice should learn the technique and basic principles during surgery by assisting an expert that is willing to point out the elements. Adopting the necessary techniques will be a gradual approach from easier to increasingly complex pathology. To always have fixed retractors mounted and ready to use is a good practice to not put the patient at risk should a situation occur where the surgeon was more confident with a retractor in place; plus, it helps to improve one's own confidence, as no time is lost should the need for fixed retraction arise.

Naturally it is easier to begin working on superficial pathology that is not covered by parenchyma. Good positioning uses gravity to move a lobe caudally in order to open up a corridor (e.g., pathology side facing downward). Less instruments will be used more and more intensely allocating them more than one function. So, dynamic retraction is possible with the shaft of an instrument for more macroscopic shift of tissue or in a true microsurgical manner by using the instrument's tip. For instance, one will find that a scissor is not restricted to the use of cutting tissue.

A completely clean operative field with only low-profile attachments, restriction to fewer instruments, less cotton patties (one displayed at the instrument trajectory is usually enough), and less of anything obstructing the view is key to increased maneuverability in the operative corridor especially so for cerebrovascular surgery with the need for clip application. Even with a fixed retractor technique, creation of a "cottonoma" around the pathology never is a good idea.

It is easier to protect uncovered tissue. To focus on deep-seated areas implies the use of a high (or the highest) zoom factor. At the same time, superficial tissue that is not in focus must not be put at risk or maltreated by untoward instrument contact and pressure (e.g., instrument shafts).

Precise and bloodless arachnoid and sulcal opening by different means and maneuvers is key to open spaces that will unfold into dissection corridors.

The "water dissection technique" is a great way to open closed space perfectly clean and tissue-protecting [7].

\subsection{Suggestion to Avoid Complication}

- Be aware of attempting a complete retractorless approach on your own if you are technically not ready yet.

- Be aware of false ambition, in a situation, where a retractor would enhance your surgery and simply put would be the better choice for the patient's brain.

- Be aware of created dissection corridors that do not allow for sufficient view. "Sufficient view" is quite relative and depends to a part also on already existing expertise.

- Be aware of making compromises to work retractorless. 
- Retractorless dissection technique alone does not do the trick; it is the involved tissue sparing and bloodless clean and very meticulous necessary technique that is necessary for work without the use of a fixed retractor that will lead to a save, more focused, and fast tissue sparing flow of surgery.

\subsubsection{Vascular Pathology with Higher Likelihood to Need Fixed Retraction Within Transition Process}

- MCA-Depending on Sylvian fissure configuration and opening, brain edema and atrophy will require temporary fixed retraction for the non-experienced to prevent the lobes falling back into the dissection corridor.
- AComA aneurysms-Will be difficult to do without fixed retraction and mini-craniotomy if not already experienced with retractorless dissection technique.

\subsection{Approach Examples}

The figure series shows examples of created dissection corridors for different vascular pathology and approaches.

\subsubsection{Anterior Circulation}

\subsubsection{Lateral Supraorbital}

(Fig. 30.5)

This is our preferred approach for anterior circulation pathology. A craniotomy cut flush to the
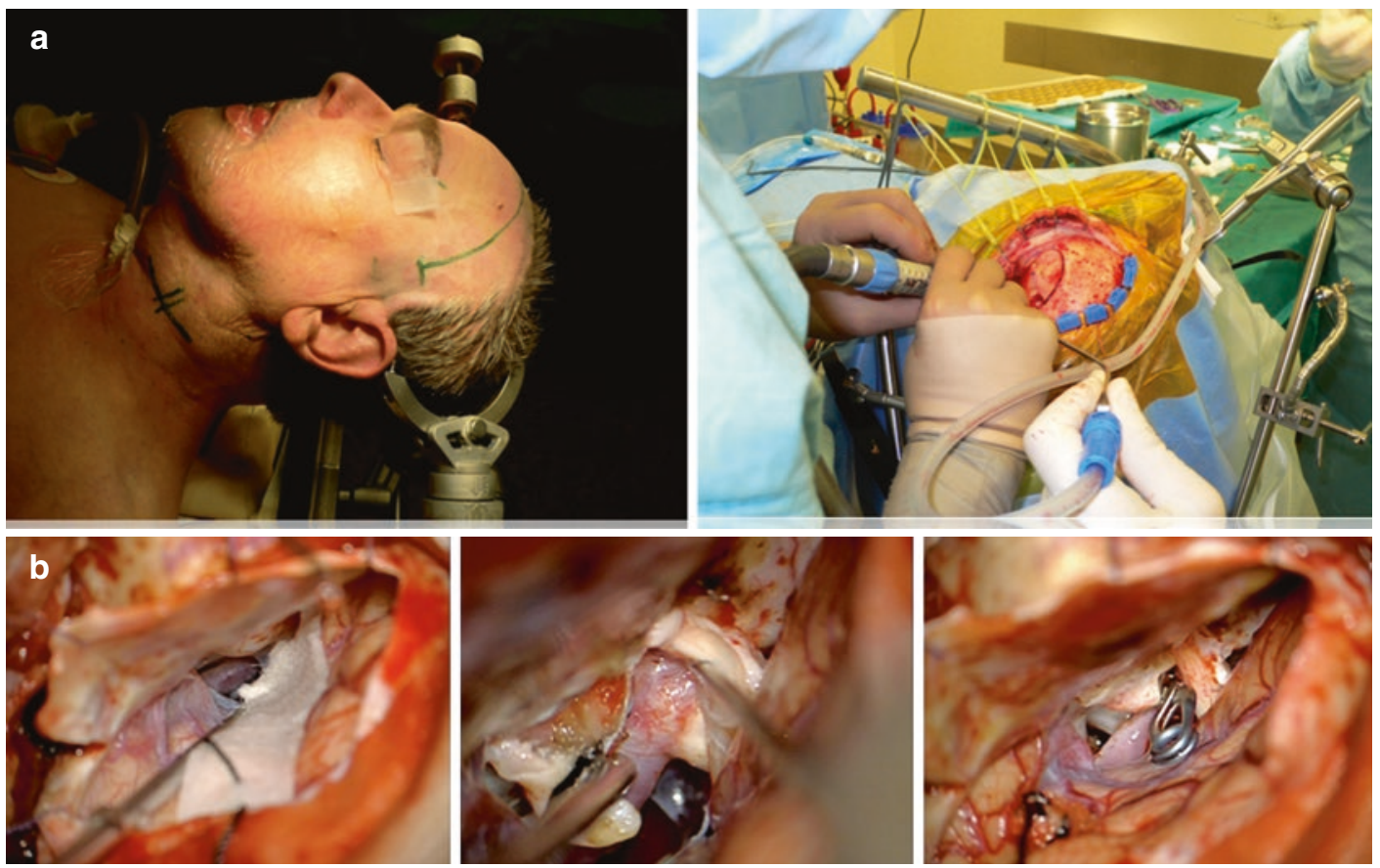

Fig. 30.5 Lateral supraorbital approach. (a) Positioning and incision for ophthalmic artery aneurysm on the left side with head elevated and reclined only mildly turned to the right with additional skin mark for exposure of carotid artery for proximal control (left image). Right craniotomy with free surgical field and attachment bars far below surgical plane to enable free hand movements (right image). (b) Left-surgical corridor right after cistern has been opened without additional retraction due to craniotomy cut flush with frontal floor and dura pulled over the bone. Middleexposure of ICA (sucker on artery), optic nerve lifted with dissector, below and left of dissector ophthalmic artery, below and right of dissector the calcified aneurysm. Partially drilled anterior clinoid process in front and left of suction with mobilized dural lip in left corner. External dural ring already opened and optic canal partially unroofed to allow for secure optic nerve mobilization. Right-surgical site with aneurysm clipped, dural flap folded back 
cranial base, and a reflected dura pulled well over this base, opens a subfrontal corridor even before the optic cistern is opened. It is thus very easy to open the cistern right from the beginning without placement of a retractor.

\subsubsection{Sylvian Fissure (Fig. 30.6)}

Doing the opening of Sylvian fissure with full focus and fast, is a very important dissection module [8-10]. It is important to have a good orientation to not choose the wrong trajectory and get lost in the fissure. Taking into account the position of the patient head in relation to the floor (angled down) is key to not move to along the wrong trajectory. Flushing the fissure with saline intermittently to open it up is a great way to help dissection (water dissection technique after Toth; see above).

\subsubsection{Interhemispheric (Fig. 30.7)}

ACA aneurysms are classically approached interhemispherically. A corridor can be created no matter if the head is in neutral or in $90^{\circ}$ turned position. For this we do not see the need for additional lumbar drainage. The depicted example of a large thalamic cavernoma demonstrates how trajectory planning, use of navigation, and gravity can greatly help to create a minimal invasive but large (enough) corridor for removal of pathology in an eloquent area.

\subsubsection{Subtemporal (Fig. 30.8)}

Subtemporal work is manageable without retraction. Retractors placed subtemporally tend to create marked pressure effects even if placed extradural. Key to retractorless at this location is placement of a lumbar drain and ample CSF drainage $(30-60 \mathrm{ml})$. This is the only approach, where we use routine lumbar drainage.

\subsubsection{Posterior Circulation}

\subsubsection{Retrosigmoid Suboccipital}

(Fig. 30.9)

For retrosigmoid approaches we prefer the patient at supine position and the head turned slightly. After the cisterna magna has been opened, there is ample room to dissect. There is enough space to drill the petrous bone as shown or remove large pathology. Large pathology and frequent use of table repositioning are made in dependence on dissection trajectory (e.g., brain stem/cerebellum table is angled toward floor, toward tentorium table is angled up, and toward petrous bone table is rotated in longitudinal axis).
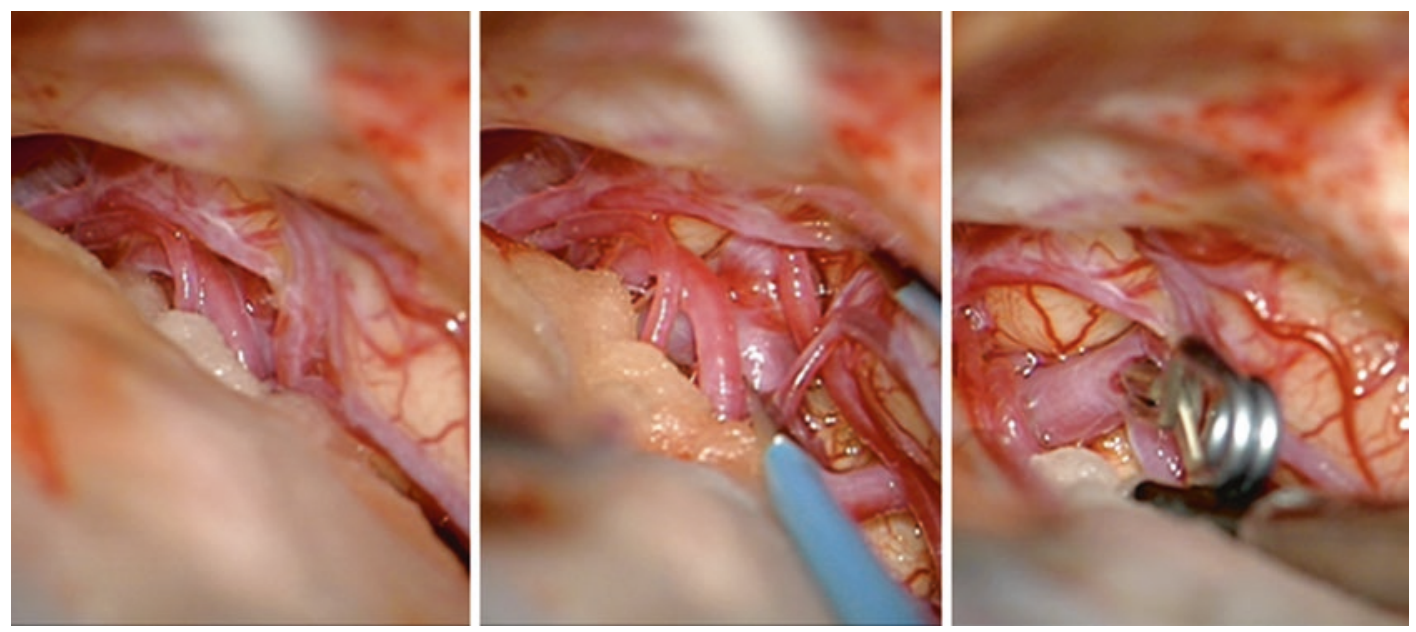

Fig. 30.6 Trans-Sylvian approach. Focused opening and clipping of left Sylvian fissure via lateral supraorbital approach for small, broad-based media aneurysm 

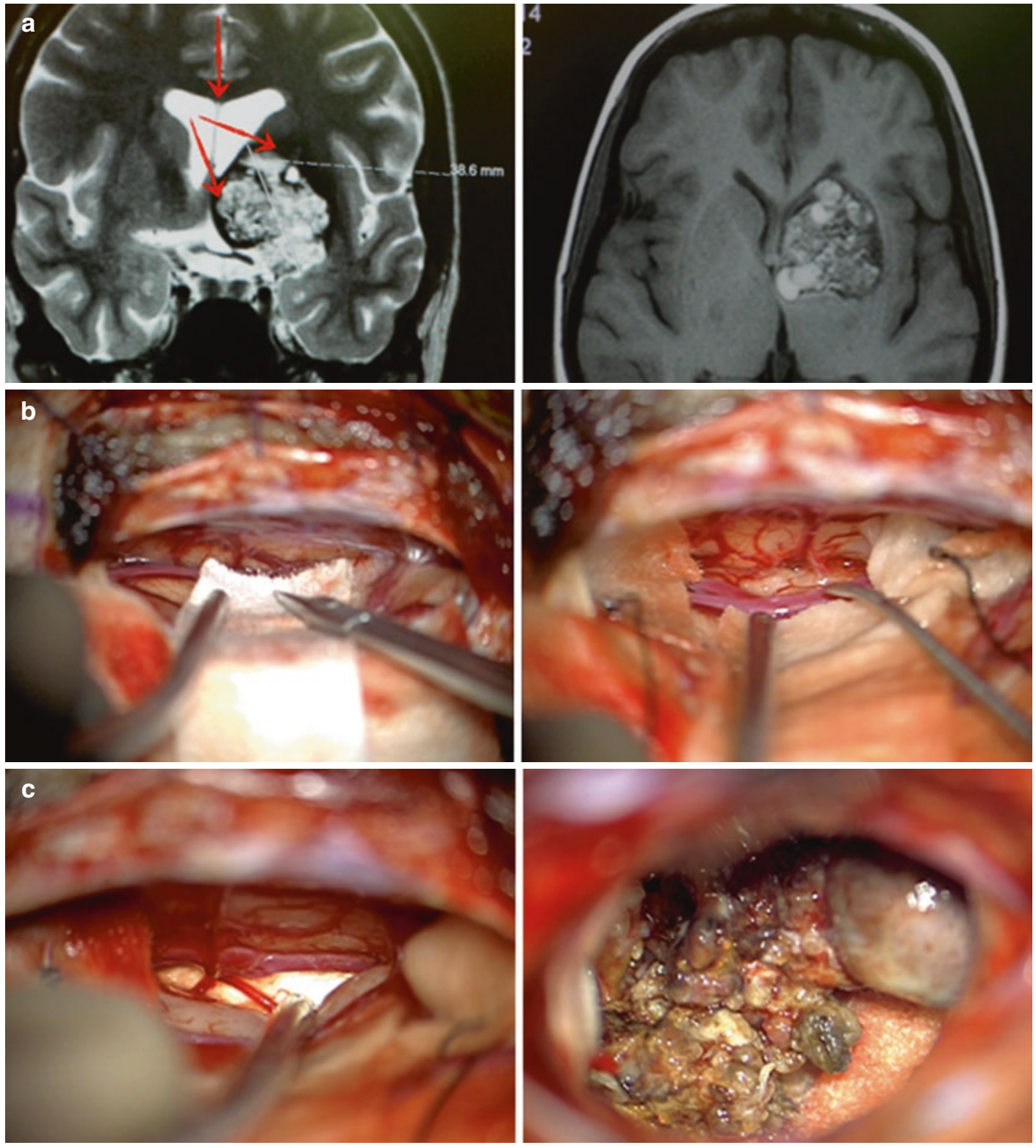

Fig. 30.7 Interhemispheric approach. Depicted is an interhemispheric approach with a contralateral transcallosal route for a basal ganglia cavernoma. (a) Left-coronal view of left basal ganglia cavernoma and interhemispheric-contralateral-transcallosal route in order to obtain a direct view on the cavernoma once the right frontal lobe recedes back by gravity. Right-axial view of cavernoma. (b) Left-patient is placed supine-semilateral, head is turned $45^{\circ}$ with temporal squama parallel to floor and raised in order to erect the falx from a "parallel-to-floor" position to around $60^{\circ}$ angled to floor. A mini-craniotomy traversing the sagittal sinus was fashioned. The dura can be seen pedicled into direction of the sagittal sinus and gently pulled back by dural stitches in

order to allow a direct view to the falx. Adhesions have been released, and the right frontal lobe did already recede underneath the dura. Right-dissection already progressed deeper to below falx level, as can be seen by contralateral frontal lobe. (c) Left-glistening white of corpus callosum underneath suction tip. Right-partially dissected cavernoma after some coagulation for size shrinking, placed within ventricle depicting typical mulberry appearance. In order to reach different parts of this large process, the trajectory is systematically changed in dependence on part of cavernoma addressed. This is reached by changing patient/OR table and microscope head position alike (e.g., Trendelenburg/reversed Trendelenburg, rotation around longitudinal axis of patient) 

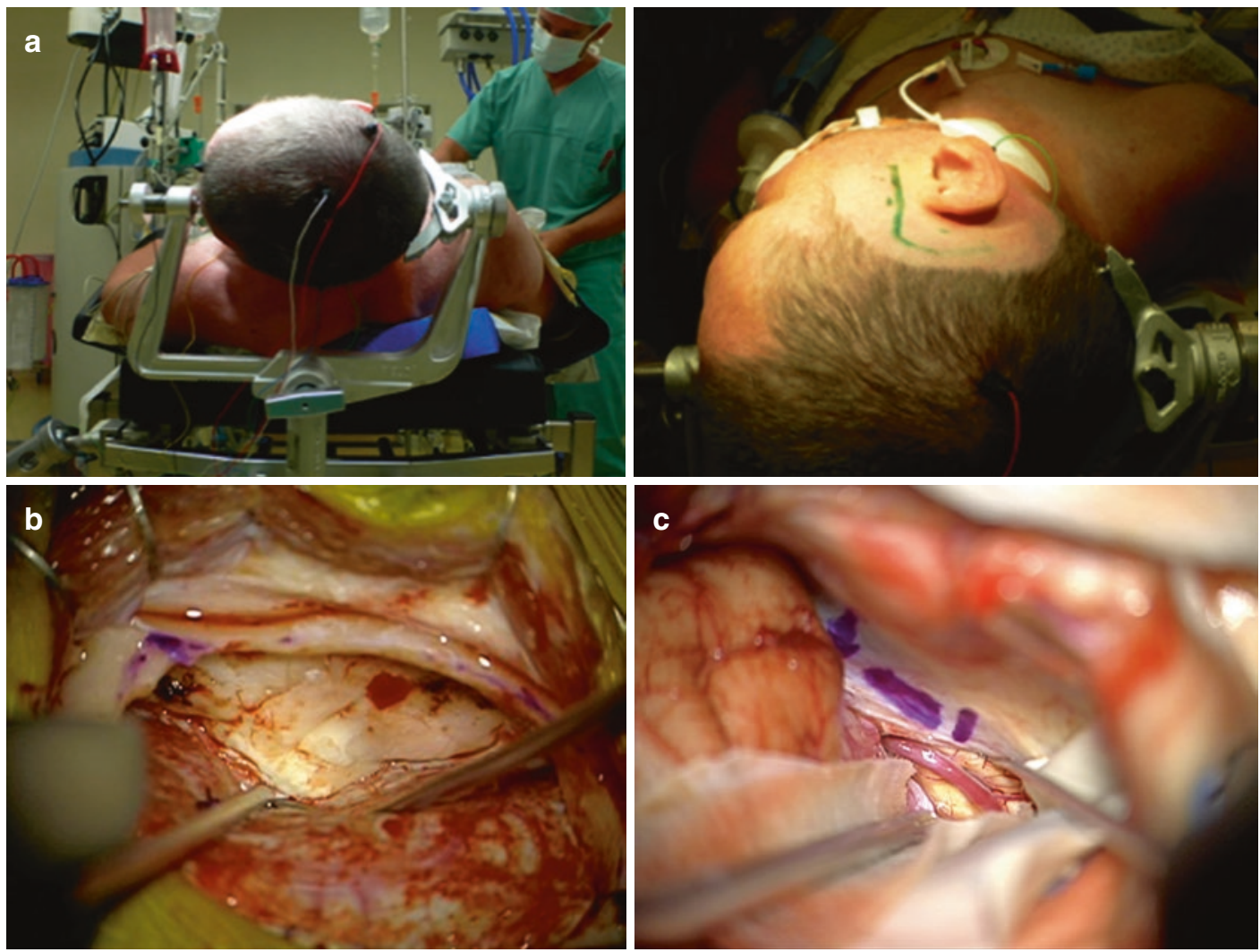

Fig. 30.8 Subtemporal approach. Easily, obtainable space in a subtemporal approach. Prerequisite is prior lumbar drainage. (a) Above-patient position with head elevated above heart level, temporal squama parallel to floor by placement of right shoulder bolster (blue) and turning the head $60-70^{\circ}$ to the left. Below-hockey stick/L-shaped/ straight skin incision mark; incisions are variable depending on planned shape of craniotomy (more horizontal width vs height) accentuating either vertical or horizontal part. (b) Extradural view on petrous bone exposing ante-

\subsection{Expert Opinion}

A retractorless technique forces the surgeon to make full use of the known microsurgical techniques in a very meticulous way. At the same time, it opens the mind to avoid any unnecessary adjuncts of surgery and leads to a very target directed way of surgery circumventing unnecessary moves. Every surgical maneuver and movement should lead to a dissection progress. The surgeons' hands stay within the operative site, and frequent in and out movements of the hands are to be avoided.

rior and dorsal rim and beginning of petrosal tip without retraction, after proper positioning, drainage of $50 \mathrm{ml} \mathrm{CSF}$, and low mini-craniotomy cut close to floor (on purpose not completely flush, for pathology approached in this case). (c) Subtemporal view on ponto-mesencephalic vein for extirpation of ponto-mesencephalic cavernoma. Narrow dark line above vein from middle to right arises from cavernoma gleaming through. Sucker on cotton patty placed on subtemporal cortex. Dissector on tentorial edge, tentorium marked with blue pen

As a result, the dissection progress of somebody used to work microsurgically without any retractor is usually very straightforward. Brute maneuvers need to be avoided at all times. Retractorless advancement only makes sense if it is less traumatic as compared to a continuously placed retractor. Overall it comes down to rigorously using the additional space that is given by opening the neighboring cisterns, gravity, and bone reduction. It raises the awareness for tissue control and avoids any additional adjuncts in the operative field. For example, surgeon put an unnecessary carpet of cotton patties, that interferes with clip application. 

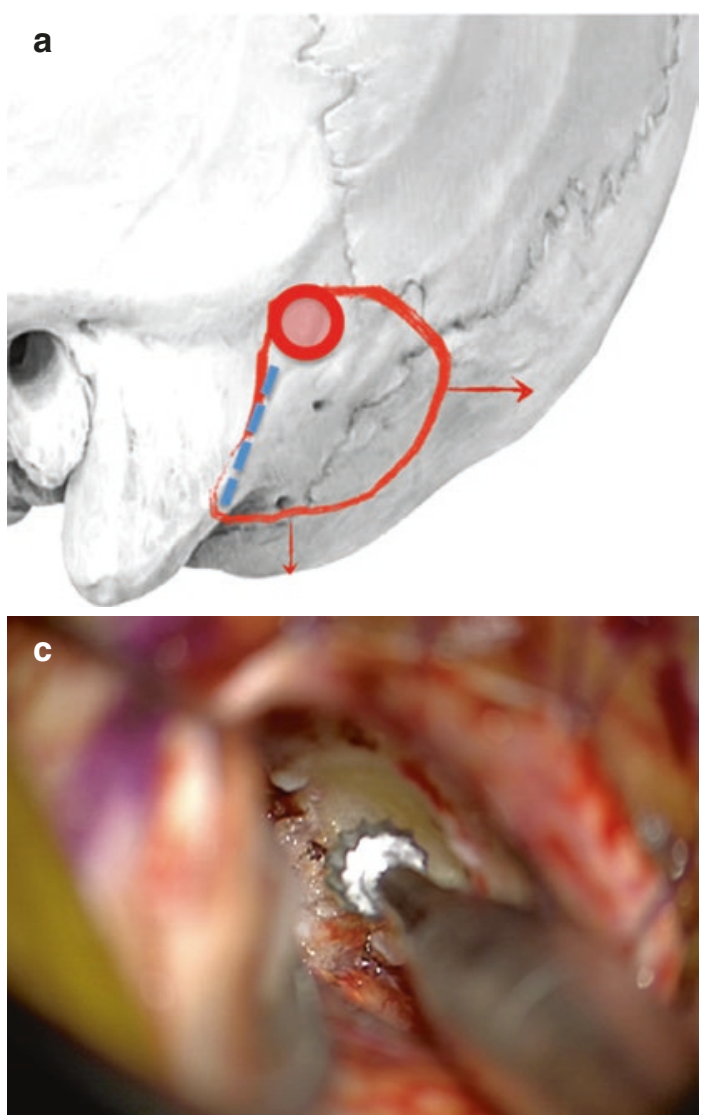

Fig. 30.9 Retrosigmoid approach. A retrosigmoid minicraniotomy is depicted with patient at supine position, and the head is turned toward the contralateral side. Proper positioning and initial CSF drainage from the cisterna magna readily open a dissection corridor even with large posterior fossa pathology. Corridors are usually large enough to also enable for microsurgical drilling. (a) Drawing shows osteoplastic craniotomy with possible extensions (arrows) depending on pathology. We prefer a burr hole placed directly into the transverse-sigmoid angle, straddling normal dura to be able to envision sinus and dura. The burr hole placement is greatly helped by neuro-navigation. Curved line depicts craniotomy; straight line is created by craniotomy drill without covering shoe. This creates a tear-off edge. Along this line, we
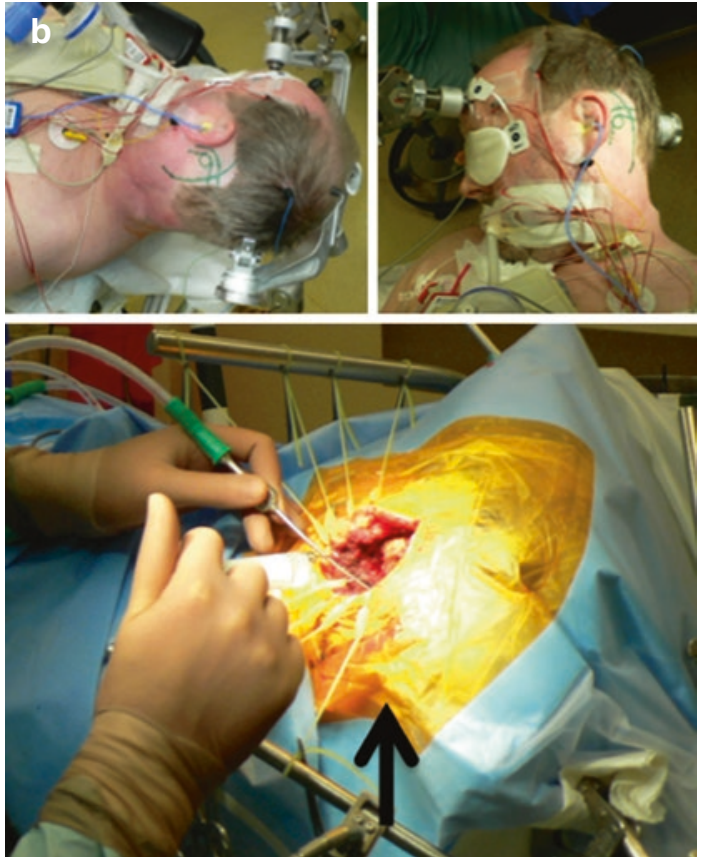

lever the bone flap out, in order to not risk a sinus tear. This technique is fast and prevents tedious drilling and at the same time minimizes bone loss. The bone flap is reinserted after dura closure and fixed with mini-plates and screws. (b) Positioning of patient with head elevated above heart level turned $60-70^{\circ}$ and inclination toward the contralateral shoulder. C-shaped retroauricular incision. Arrow depicts position of surgeon once dura is opened. Dissection is downward (in contrast to a sitting or semisitting position). Forearms can easily be placed on an armrest with the surgeon remaining seated. (c) A magnified surgeon's view with this kind of retrosigmoid approach, which demonstrates how a conventional drill can be safely inserted to work on the petrous bone 
The other real merit is to use mouth and feet for all the movements that usually would be done with the hands. A surgeon used to mouthpiece and foot control adjusts microscope head, focus, and zoom subcortically. An absolute pristine surgical field and removal of anything unnecessary are prerequisites. It surely helps to only use attachments that are low profile and do not interfere with the hands. Positioning and use of "gravity retraction" obtain new meaning.

Retractorless dissection can be adopted by making full use of all known neuromicrosurgical methods and principles.

The immanent necessities for retractorless neurosurgery pave the way for a far more effective and efficient and bloodless dissection technique that will be very tissue sparing.

\section{References}

1. Andrews RJ, Muto RP. Retraction brain ischaemia: cerebral blood flow, evoked potentials, hypotension and hyperventilation in a new animal model. Neurol Res. 1992;14(1):12-8.

2. Dashti R, Hernesniemi J, Niemelä M, Rinne J, Lehecka M, Shen $\mathrm{H}$, et al. Microneurosurgical management of distal middle cerebral artery aneurysms. Surg Neurol. 2007;67(6):553-63.

3. Dashti R, Hernesniemi J, Niemelä M, Rinne J, Porras M, Lehecka M, et al. Microneurosurgical management of middle cerebral artery bifurcation aneurysms. Surg Neurol. 2007;67(5):441-56.

4. Dashti R, Rinne J, Hernesniemi J, Niemela M, Kivipelto L, Lehecka M, et al. Microneurosurgical management of proximal middle cerebral artery aneurysms. Surg Neurol. 2007;67(1):6-14.

5. Hernesniemi J, Ishii K, Niemela M, Smrcka M, Kivipelto L, Fujiki M, Shen H. Lateral supraorbital approach as an alternative to the classical pterional approach. Acta Neurochir Suppl. 2005;94:17-21.

6. Kivisaari RP, Salonen O, Ohman J. Basal brain injury in aneurysm surgery. Neurosurgery. 2000;46(5):10704; discussion 1074-6.

7. Kivisaari RP, Salonen O, Servo A, Autti T, Hernesniemi J, Ohman J. MR imaging after aneurysmal subarachnoid hemorrhage and surgery: a long-term follow-up study. AJNR Am J Neuroradiol. 2001;22(6):1143-8.

8. Molyneux A. International Subarachnoid Aneurysm Trial (ISAT) of neurosurgical clipping versus endovascular coiling in 2143 patients with ruptured intracranial aneurysms: a randomised trial. Lancet. 2002;360(9342):1267-74.

9. Nagy L, Ishii K, Karatas A, Shen H, Vajda J, Niemela $\mathrm{M}$, et al. Water dissection technique of Toth for opening neurosurgical cleavage planes. Surg Neurol. 2006;65(1):38-41.

10. Spetzler RF, McDougall CG, Albuquerque FC, Zabramski JM, Hills NK, Partovi S, et al. The barrow ruptured aneurysm trial: 3-year results. J Neurosurg. 2013;119(1):146-57.

Open Access This chapter is licensed under the terms of the Creative Commons Attribution 4.0 International License (http://creativecommons.org/licenses/by/4.0/), which permits use, sharing, adaptation, distribution and reproduction in any medium or format, as long as you give appropriate credit to the original author(s) and the source, provide a link to the Creative Commons license and indicate if changes were made.

The images or other third party material in this chapter are included in the chapter's Creative Commons license, unless indicated otherwise in a credit line to the material. If material is not included in the chapter's Creative Commons license and your intended use is not permitted by statutory regulation or exceeds the permitted use, you will need to obtain permission directly from the copyright holder. 